\title{
Hyaline fibrous involution of breast lobules: a histologic finding associated with germline $B R C A$ mutation
}

\author{
Hee Eun Lee ${ }^{1}$ Muhammad Arshad ${ }^{2} \cdot$ Rushin D. Brahmbhatt ${ }^{2} \cdot$ Tanya L. Hoskin $^{3}$ - Stacey J. Winham (1) ${ }^{3}$. \\ Marlene H. Frost ${ }^{4} \cdot$ Derek C. Radisky $^{5} \cdot$ Lori A. Denison $^{6} \cdot$ Amy C. Degnim $\mathbb{D}^{2} \cdot$ Daniel W. Visscher $^{1}$
}

Received: 15 March 2018 / Revised: 16 January 2019 / Accepted: 19 January 2019 / Published online: 17 April 2019

(c) United States \& Canadian Academy of Pathology 2019

\begin{abstract}
We describe the histology and the frequency of a histologic entity that we term "hyaline fibrous involution", which is characterized by symmetric and regular deposition of basal lamina-like periacinar hyaline material in association with atrophic epithelium, in breast samples from patients with either benign breast disease or germline BRCA mutation. Women with germline $B R C A$ mutation $(n=93)$ who underwent prophylactic mastectomy (BRCA group) were compared to an age-matched sample of women who underwent biopsy for benign breast disease $(n=93)$. Median age was 45 years (range, 25-72 years). A single H\&E section of each subject's benign breast tissue was reviewed. The total number of terminal duct lobular units and the number of terminal duct lobular units with hyaline fibrous involution were recorded for each case. The presence of any hyaline fibrous involution lobules and the within-sample proportion of hyaline fibrous involution lobules relative to total lobules were compared between groups. Presence of any hyaline fibrous involution was significantly more frequent in the BRCA group compared to the benign breast disease group, $47 \%$ vs. $15 \%(p<0.0001$, adjusted for total lobules). In women with any hyaline fibrous involution lobules, these unusual lobules were similarly rare in both groups, with median proportion of hyaline fibrous involution-positive lobules relative to all lobules of 0.03 in BRCA specimens $(n=44)$ and 0.03 in the benign breast disease group $(n=14)$. Within the BRCA group, frequency of any hyaline fibrous involution present was significantly higher in the perimenopausal age group (45-55 years: 63\%) compared to other age groups ( $<45$ years, $44 \%$; $>55$ years, $15 \% ; p=0.05$ and $p=0.02$, respectively). Increased presence of hyaline fibrous involution in the setting of $B R C A$ mutation suggests that it may represent a pathologic entity, possibly reflecting abnormal involution or an abnormal response to DNA damage.
\end{abstract}

This work has been, in part, presented at the Annual United States and Canadian Academy of Pathology meeting in March, 2016 in Seattle, WA, USA.

Daniel W. Visscher

Visscher.Daniel@mayo.edu

1 Department of Laboratory Medicine and Pathology, Mayo Clinic, Rochester, MN, USA

2 Department of Surgery, Mayo Clinic, Rochester, MN, USA

3 Department of Health Sciences Research, Mayo Clinic, Rochester, MN, USA

4 Department of Medical Oncology, Mayo Clinic, Rochester, MN, USA

5 Department of Cancer Biology, Mayo Clinic, Rochester, MN, USA

6 Department of Information Technology, Mayo Clinic, Rochester, MN, USA

\section{Introduction}

Lobular involution is an age associated physiological process in which terminal duct lobular units undergo progressive loss of cell populations over the course of decades, leading eventually to atrophy [1]. Involution results in terminal duct lobular units with fewer numbers of small acini that have flattened and inconspicuous epithelium along with loss of intralobular stroma [1]. There is evidence that delayed or interrupted involution is associated with increased risk for breast cancer development in women with benign breast disease [2, 3].

BRCA genes act as tumor suppressor genes and mediate double-stranded DNA break repair [4-6]. Pathogenic germline mutations in BRCA1 and BRCA2 are associated with a markedly increased susceptibility to breast carcinomas: female carriers of mutations in either gene have a lifetime risk of breast cancer of at least $50 \%$ [4, 5, 7]. 
Although the pathological features of breast cancers arising in the setting of BRCA germline mutations have been welldescribed, little is known about premalignant or benign alterations in the breast tissue of these women. Specifically, terminal duct lobular unit involution has not been studied.

This study describes a novel terminal duct lobular unit alteration that is characterized by periacinar deposition of basal lamina-like hyaline material in association with atrophic-appearing epithelium. This lobular alteration is similar to age-related lobular involution in terms of acinar loss, but different in the sense that it shows expansion of intralobular extracellular matrix by what appears to be pathological fibrosis. We have termed this lobular alteration 'hyaline fibrous involution' because of the hyalinized fibrosis in lobules demonstrating decreased diameter relative to (preinvolutional) normal lobules. We hypothesize that hyaline fibrous involution may reflect a pathological form of terminal duct lobular unit involution. The present study was undertaken to evaluate the possible association of hyaline fibrous involution with $B R C A$ mutation status.

\section{Materials and methods}

\section{Study population}

Following approval by the Institutional Review Board of the Mayo Clinic, archival $\mathrm{H}$ and $\mathrm{E}$ stained sections were obtained from the Mayo Clinic Tissue Registry, including 93 women with germline deleterious BRCA mutations who underwent prophylactic mastectomies (1993-2012). A comparison group of 93 age-matched women was obtained from the Mayo Clinic Benign Breast Disease Cohort, which is a large, well-described cohort of $\sim 13,538$ women who underwent breast biopsy with benign findings from 1967-2001, including radiologically guided core needle biopsies starting in 1994 [8]. In this group, the $B R C A$ mutation status is unknown. The benign breast disease samples for this study were restricted to the 1982-2001 portion of the benign breast disease cohort to make the tissue age more similar to that of the BRCA samples. A separate normal comparison group of 86 age-matched women who underwent reduction mammoplasty at Mayo Clinic Rochester in 1995-2001 was collected from Mayo Clinic files and archival $\mathrm{H}$ and $\mathrm{E}$ stained sections were obtained from the Mayo Clinic Tissue Registry.

\section{Histologic review}

For each subject, we reviewed all available slides and chose a representative one with the highest number of terminal duct lobular units. The selected representative benign tissue sections were examined to enumerate the total number of terminal duct lobular unit and the total number of terminal duct lobular unit with hyaline fibrous involution. Hyaline fibrous involution was defined as periacinar ring-like, regular deposition of basal lamina-like hyaline material in association with atrophic epithelium (Figs. 1 and 2). If a terminal duct lobular unit showed any hyaline fibrous involution regardless of the extent of involvement, it was called a "hyaline fibrous involution-positive lobule." Hyaline fibrous involution-positive lobules were further categorized as diffusely or focally involved. A terminal duct lobular unit involved by hyaline fibrous involution in $\geq 50 \%$ of the total lobule area was designated as a "hyaline fibrous involution-diffuse lobule" (Fig. 2b), whereas hyaline fibrous involution in $<50 \%$ of the lobule area was designated as a "hyaline fibrous involution-focal lobule" (Fig. 2d). The total numbers of terminal duct lobular units present on the slide, and the numbers of hyaline fibrous involution-diffuse lobules and hyaline fibrous involution-focal lobules, were evaluated and recorded separately in each case.

\section{Associations with clinical and pathologic features and follow-up}

Associations of hyaline fibrous involution lobules with patient age and histologic age-related involution ("none" vs. "partial" vs. "complete") were evaluated. Age-related involution was classified as none if $<25 \%$ of normal lobules were involuted, as partial if $25-75 \%$ were involuted, and complete if $>75 \%$ were involuted. Follow-up for breast cancer events in the benign breast disease group only was obtained through comprehensive Mayo medical records and a study-specific questionnaire. The presence of any hyaline fibrous involution lobule(s) and the within-sample proportion of hyaline fibrous involution lobules relative to the total number of lobules were compared between groups.

\section{Statistical analysis}

Number of hyaline fibrous involution lobules was analyzed as a count variable using a negative binomial regression model with the logarithm of the total number of lobules as offset and a covariate for group (BRCA vs. benign breast disease). Multivariable models additionally adjusting for degree of age-related lobular involution were also fit, as were models assessing impact of factors such as type of mutation (BRCAl vs. BRCA2) within the BRCA subset. For the presence of any hyaline fibrous involution lobules within the sample, logistic regression adjusted for total lobules was used to compare groups. Negative binomial regression models were fit using the glm.nb function from the MASS library [9] for R (www.r-project.org). Among the benign breast disease subjects, the association of hyaline fibrous involution with future breast cancer events was 

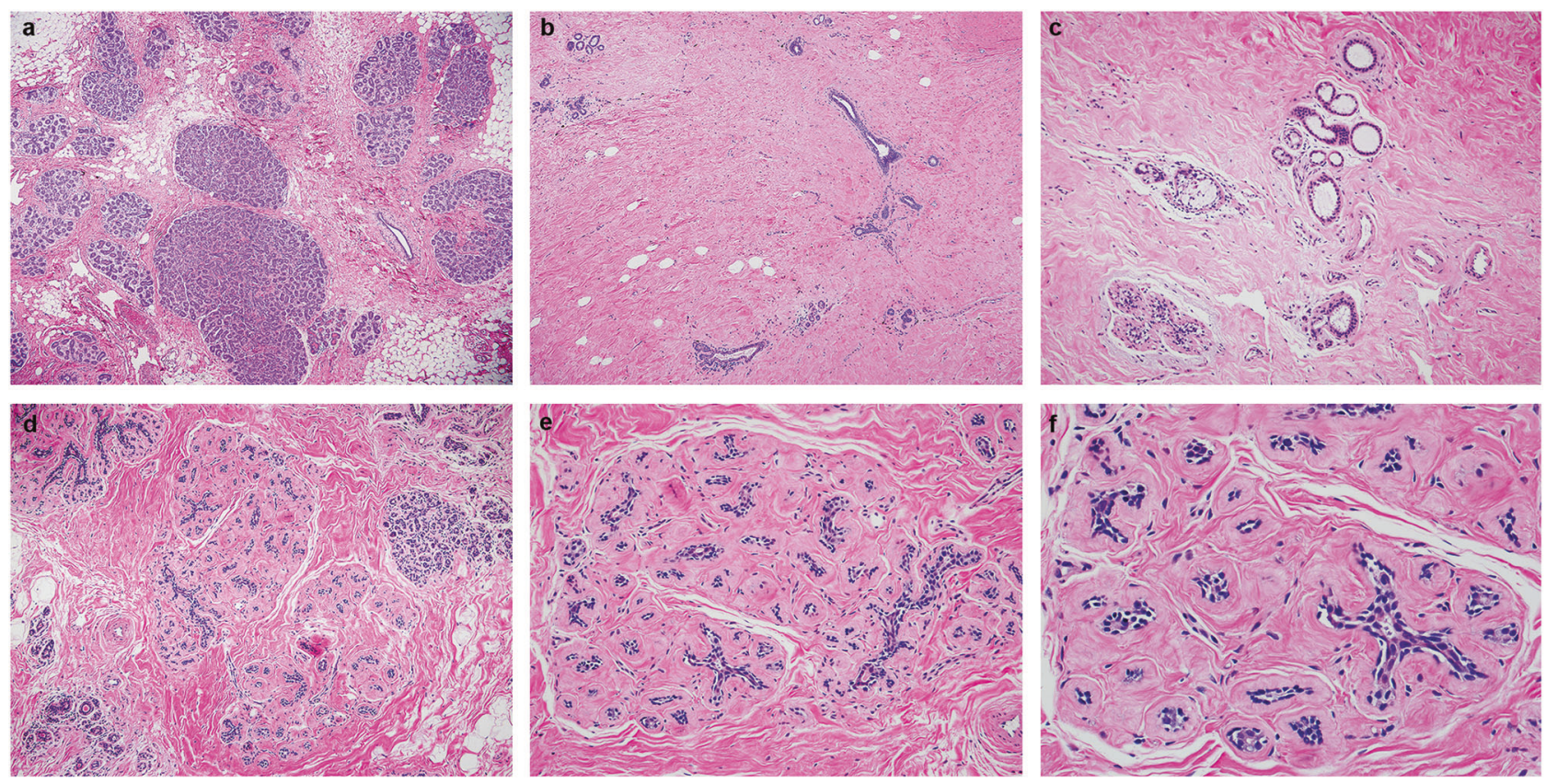

Fig. 1 Comparison between age-related lobular involution and hyaline fibrous involution. a Non-involuted breast parenchyma of a premenopausal woman. b, c Age-related lobular involution characterized by terminal duct lobular units with fewer numbers of small acini that have flattened and inconspicuous epithelium along with loss of intralobular stroma. d-f Hyaline fibrous involution characterized by symmetric and regular, periacinar ring-like deposition of basal lamina-like hyaline material in association with atrophic epithelium assessed with Fine and Gray competing risks regression using the cmprsk package [10]. $p$-values $<0.05$ were considered statistically significant.

\section{Histochemical and immunohistochemical staining}

We selected 19 samples with hyaline fibrous involution (14 BRCA and five benign breast disease), for immunostains to investigate the composition of the hyalinized basal laminalike material in the hyaline fibrous involution lobules. A representative block was chosen from each case to stain with Masson-trichrome, elastin, and collagen type IV. Immunohistochemical stains were performed on four micron sections cut from formalin-fixed, paraffin-embedded blocks, which were placed on "charged" slides; slides were dried and melted in a $62{ }^{\circ} \mathrm{C}$ oven for twenty minutes. Slides were placed on a Ventana BenchMark XT (Ventana Medical Systems Inc.) for staining. Immunohistochemistry was performed with a mouse monoclonal antibody to collagen IV (clone CIV22; 1/50 dilution) (Dako, Carpinteria CA). The staining protocol includes online deparaffinization, HIER (Heat Induced Epitope Retrieval) with Ventana Cell Conditioning 1 Mild, primary antibody incubation for 32 minutes at $37^{\circ} \mathrm{C}$. Antigen-antibody reactions are visualized using the Ventana UltraviewTM Universal DAB Detection Kit. Counterstaining is performed online using Ventana Hematoxylin II for $8 \mathrm{~min}$, followed by bluing reagent for $4 \mathrm{~min}$. Slides are removed from the stainer, dehydrated, and coverslipped for microscopic examination.

\section{Results}

\section{Patient sample}

Among 93 women with $B R C A$ mutations, 58 (62\%) were BRCA1 mutations and 35 (38\%) were BRCA2 mutations. Mastectomies were bilateral prophylactic in $33(36 \%)$ and contralateral prophylactic in $60(65 \%)$ patients with concurrent or prior history of breast cancer on the opposite side. Median age was 45 years with a range from 25 years to 72 years for both BRCA and benign breast disease groups. Although the two groups were age-matched, they did differ significantly on degree of age-related lobular involution $(p=0.001)$ with complete involution in $29 \%$ of BRCA subjects vs. $13 \%$ of the benign breast disease subjects, no involution in $12 \%$ of BRCA subjects vs. $31 \%$ of the benign breast disease subjects, and partial involution for similar percentages (59\% of BRCA, $56 \%$ of benign breast disease).

\section{Comparing hyaline fibrous involution measures between BRCA and benign breast disease groups}

The median total number of lobules per sample was 106 (range, 7-480) in the BRCA group prophylactic 
Fig. 2 Photomicrographs of hyaline fibrous involution. a Low-magnification of breast parenchyma with hyaline fibrous involution. b A terminal duct lobular unit involved by hyaline fibrous involution in $\geq 50 \%$ of the total lobule area was designated as a "hyaline fibrous involution-diffuse lobule". c High magnification of the hyaline fibrous involution seen in b. d A terminal duct lobular unit involved by hyaline fibrous involution in $<50 \%$ of the total lobule area was designated as a "hyaline fibrous involution -focal lobule". e, f A terminal duct lobular unit was diffusely involved by hyaline fibrous involution, with symmetric and regular, periacinar ring-like deposition of basal lamina-like hyaline material (e), which was stained blue in Massontrichrome stain, suggestive of collagen type I (f)
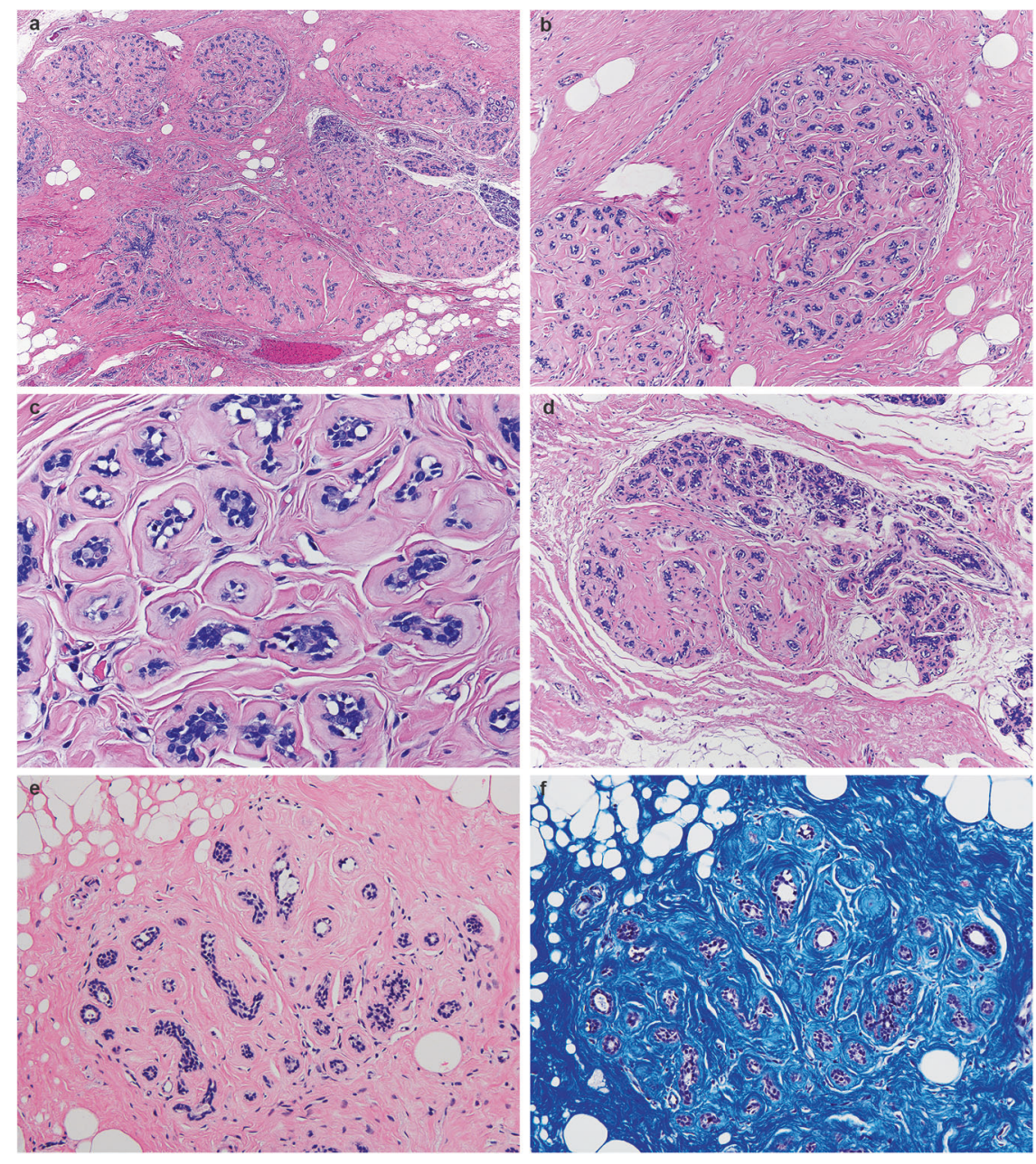

mastectomy tissues and median 62 (range, 2-321) in the benign breast disease group benign biopsy tissues, $p<$ 0.001. Presence of any hyaline fibrous involution lobule(s) was significantly more frequent in the BRCA samples compared to the benign breast disease samples, 47\% (44/93) vs. $15 \%$ (14/93) (Fig. 3). After adjusting for the total number of lobules in each sample, the odds ratio for the presence of any hyaline fibrous involution lobule(s) in the sample was 4.5 (95\% CI: 2.2-9.2, $p<0.001$ ) for BRCA vs. benign breast disease samples. Considering the number of hyaline fibrous involution lobules in the BRCA group, the within-sample proportion of hyaline fibrous involutionpositive lobules relative to total lobules was mean 0.04 (SD 0.08 ), while hyaline fibrous involution-diffuse lobules and hyaline fibrous involution-focal lobules had mean proportions of 0.03 (SD 0.07) and 0.01 (SD 0.02), respectively. In the benign breast disease group, the mean proportion of positive for hyaline fibrous involution was 0.0076 (SD 0.03), with mean proportion hyaline fibrous involutiondiffuse and hyaline fibrous involution-focal lobules of 0.0053 (SD 0.02) and 0.0023 (SD 0.01), respectively. The count of hyaline fibrous involution lobules relative to total lobules was statistically different between the BRCA and benign breast disease subjects after adjusting for total number of lobules in the sample (hyaline fibrous involution-positive lobules, $p<0.001$; hyaline fibrous involution-diffuse lobules, $p<0.001$; and hyaline fibrous involution-focal lobules, $p=0.003$ ). These differences each remained statistically significant after adjustment for degree of age-related lobular involution. In the subset who did have any hyaline fibrous involution (44/93 BRCA and 14/93 benign breast disease samples), the percent of hyaline fibrous involution lobules relative to total lobules was low in both groups: median 0.03 (range, $0.004-0.45$ ) for BRCA specimens and median 0.03 (range, 0.01-0.18) for benign breast disease, which was not significantly different $(p=0.14 ;$ Table 1$)$.

\section{Comparing hyaline fibrous involution measures between BRCA or benign breast disease and reduction mammoplasty groups}

Hyaline fibrous involution was assessed on a separate normal comparison group comprised of 86 reduction 


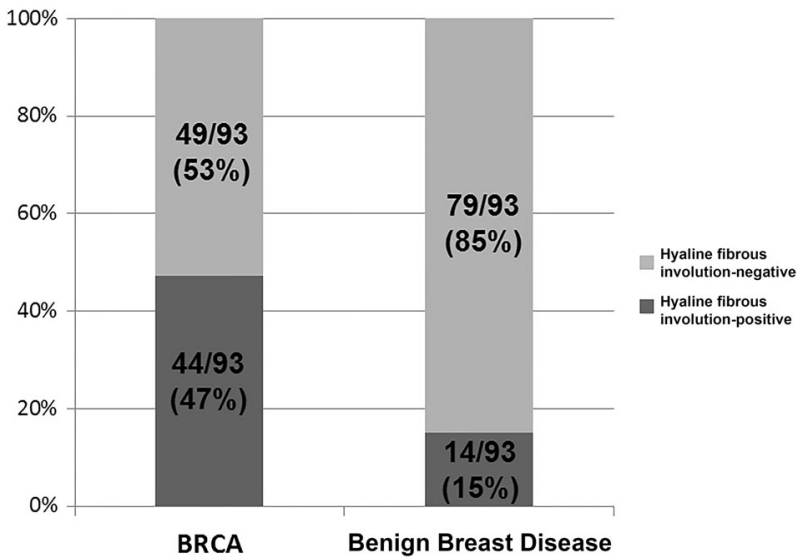

Fig. 3 Frequency of hyaline fibrous involution in the BRCA and benign breast disease groups. Forty-four of 93 cases (47\%) in BRCA group showed hyaline fibrous involution as compared to 14 of 93 cases $(15 \%)$ in the benign breast disease group, which was a statistically significant difference. After adjusting for the total number of lobules in each sample, the odds ratio was 4.5 (95\% CI: $2.2-9.2, p<0.001)$ for presence of any hyaline fibrous involution for the BRCA vs. benign breast disease samples

Table 1 Comparing the proportion of lobules with hyaline fibrous involution (HFI) between groups among the subset of samples with any HFI lobules present

\begin{tabular}{llll}
\hline & BRCA $(n=44)$ & BBD $(n=14)$ & $p$-value \\
\hline $\begin{array}{l}\text { HFI-positive } \\
\text { lobules/total }\end{array}$ & $0.03(0.004-0.45)$ & $0.03(0.01-0.18)$ & 0.14 \\
lobules $^{\mathrm{b}}$ & $0.08(0.11)$ & $0.05(0.05)$ & \\
HFI-diffuse lobules/ $_{\text {total lobules }}$ b & $0.02(0-0.39)$ & $0.02(0-0.11)$ & 0.12 \\
HFI-focal lobules/ $_{\text {total lobules }}$ & $0.06(0.10)$ & $0.04(0.04)$ & \\
\hline
\end{tabular}

$B R C A$ BRCA gene-mutated group, $B B D$ benign breast disease group, $H F I$ hyaline fibrous involution

${ }^{a}$ BRCA vs. BBD; adjusted for total lobules and age

${ }^{b}$ Values represent median (minimum-maximum), mean (standard deviation)

mammoplasty sample from patients with an age distribution similar (median 44 years, range 27-77 years) to the BRCA and benign breast disease groups. These reduction mammoplasty specimens were all confirmed to have either no histologic abnormality $(16 \%)$ or only nonproliferative benign breast changes (84\%). Only $1 / 86$ $(1 \%)$ reduction mammoplasty specimens had any hyaline fibrous involution lobules represent; this incidence was significantly lower than observed for BRCA $(p<0.001)$ and benign breast disease samples $(p=0.02)$, which demonstrated the presence of at least one hyaline fibrous involution lobule in $47 \%$ and $15 \%$ of samples, respectively.
Association of hyaline fibrous involution with other variables

Within the BRCA subjects, there was no significant difference in number of hyaline fibrous involution based on $B R C A 1$ vs. $B R C A 2$ mutations $(p=0.54)$ or whether the patient was undergoing bilateral prophylactic mastectomy vs. contralateral prophylactic mastectomy $(p=0.24)$. However, the presence of any hyaline fibrous involution lobule(s) within the sample was significantly more frequent in the perimenopausal age group (45-55 years: 63\%) compared to other age groups $(<45$ years, $44 \%$; $>55$ years, $15 \%), p=0.05$ and $p=0.02$, respectively (Table 2). Also within the BRCA group, those with either partial $(p=$ $0.008)$ or complete $(p=0.01)$ age-related lobular involution showed significantly higher proportion of hyaline fibrous involution lobules compared to subjects where involution was classified as none. In the benign breast disease group, there was no apparent association between age and presence of any hyaline fibrous involution lobules or the number of hyaline fibrous involution lobules. However, the presence of any hyaline fibrous involution lobule and the number of hyaline fibrous involution-positive lobules per total lobules did differ by age-related lobular involution in benign breast disease subjects and was significantly higher for those with partial compared to those with complete and none $(p=0.03$ and $p=0.002$, respectively).

\section{Hyaline fibrous involution and breast cancer risk}

During a median of 16 years follow-up, 12 of 93 cases in the benign breast disease group developed breast cancer. On univariate analysis, presence of any hyaline fibrous involution-positive lobule(s) in women with benign breast disease showed a HR of 2.06 (95\% CI: $0.49-8.64, p=$ 0.32 ), but this effect size was attenuated when adjusted for age (HR 1.49, 95\% CI: $0.46-4.88, p=0.51$ ).

\section{Special stains}

All 14 cases stained showed that the hyalinized basal laminalike material in hyaline fibrous involution stained blue in Masson-trichrome stain (Fig. 2f), and all were negative for elastin and collagen type IV. No differences were observed between BRCA and hyaline fibrous involution samples. These findings indicate that the hyaline material in hyaline fibrous involution lobules is composed of type I collagen.

\section{Discussion}

Our data demonstrate that hyaline fibrous involution comprise a variable but relatively small proportion of total 
Table 2 Association of hyaline fibrous involution (HFI) with patient age and degree of agerelated lobular involution

\begin{tabular}{|c|c|c|c|c|}
\hline & \multicolumn{2}{|l|}{ BRCA } & \multicolumn{2}{|l|}{ BBD } \\
\hline & $\begin{array}{l}N(\%) \text { of samples } \\
\text { with any HFI- } \\
\text { positive lobules } \\
\text { present }\end{array}$ & $\begin{array}{l}\text { Mean (SD) } \\
\text { Proportion of HFI- } \\
\text { positive lobules } \\
\text { within sample }\end{array}$ & $\begin{array}{l}N(\%) \text { of samples } \\
\text { with any HFI- } \\
\text { positive lobules } \\
\text { present }\end{array}$ & $\begin{array}{l}\text { Mean (SD) Proportion } \\
\text { of HFI-positive lobules } \\
\text { within sample }\end{array}$ \\
\hline \multicolumn{5}{|l|}{ Age } \\
\hline$<45$ years & $44 \%(20 / 45)$ & $0.03(0.07)$ & $16 \%(7 / 45)$ & $0.004(0.01)$ \\
\hline $45-55$ years & $63 \%(22 / 35)$ & $0.05(0.08)$ & $14 \%(5 / 35)$ & $0.01(0.04)$ \\
\hline$>55$ years & $15 \%(2 / 13)$ & $0.04(0.12)$ & $15 \%(2 / 13)$ & $0.01(0.04)$ \\
\hline \multicolumn{5}{|c|}{ Age-related lobular involution } \\
\hline None & $36 \%(4 / 11)$ & $0.01(0.01)$ & $4 \%(1 / 27)$ & $0.0004(0.002)$ \\
\hline Partial & $53 \%(29 / 55)$ & $0.04(0.10)$ & $25 \%(12 / 49)$ & $0.01(0.04)$ \\
\hline Complete & $41 \%(11 / 27)$ & $0.04(0.07)$ & $9 \%(1 / 11)$ & $0.004(0.01)$ \\
\hline
\end{tabular}

$B R C A$ BRCA gene-mutated group, $B B D$ benign breast disease group, $H F I$ hyaline fibrous involution, $S D$ standard deviation. terminal duct lobular units in any representative breast sample. However, it is approximately threefold more likely to be present in women with $B R C A$ mutation compared to age-matched women with benign breast disease, and only rarely $(1 \%)$ seen in age-matched women with no histologic abnormalities or non-proliferative changes of the breast. Hyaline fibrous involution is seen in a small terminal duct lobular unit with atrophic epithelium, but it is histologically different from age-related lobular involution by virtue of its characteristic periacinar symmetric, regular deposition of basal lamina-like material which was found to be type I collagen by Masson-trichrome stain.

Although BRCA2-mutation associated breast cancers are not significantly different than sporadic cancers with respect to pathologic parameters, BRCAl-mutated breast cancers are typically triple-negative (ER-negative, PR-negative, HER2-negative) with high grade "basal-like" phenotype, pushing margins, and lymphocytic infiltrates [4]. Efforts to identify histopathologic features in non-neoplastic breast parenchyma that characterize or predict BRCA mutation status have yielded controversial results. Some studies indicate that high-risk (atypical) breast lesions are more common in BRCA mutation carriers than in patients with sporadic breast cancer [11-14]. Others have shown that women with $B R C A$ mutation had a lower prevalence of proliferative fibrocystic changes [15]. At the present time, there is no generally accepted finding in benign breast tissue that reliably reflects $B R C A$ mutation (i.e., in the absence of cancer).

Bayraktar et al. [16] detailed histological features of peritumoral non-neoplastic parenchyma in women with and without BRCA mutation. They assessed six histologic features such as benign proliferative breast disease with and without atypia, stromal fibrosis, lobular type, lobular contour, lobular sclerosis, and lobulitis. These authors concluded that there is no significant association between mutation status and benign histologic features in peritumoral breast parenchyma. Lobular contour, in their report, was defined as "regular" if the distinct lobular architecture was preserved with smooth borders of non-neoplastic lobules. They indicated that the prevalence of regular lobular contour was slightly higher in the $B R C A$-mutated group than in the $B R C A$-wild group (61\% vs. $52 \%$ ) although it was not statistically significant. Based on their histologic description and microphotograph published, we believe at least a part of the cases with regular lobular contour might represent hyaline fibrous involution.

Russo et al. [17] have described three "developmental patterns" of breast lobules. One of them (Lob 1 in their terminology) is characterized by clusters of 6-11 "ductules" per lobule, with intralobular fibrosis and hyalinization. These authors showed that Lob 1 pattern is more common in postpubertal nulliparous women and patients with $B R C A$ mutation (regardless of parity). They suggested that Lob 1 pattern represents a "failure of differentiation" and is associated with increased risk of breast cancer. Based on the photomicrograph published in that paper, we believe that their Lob 1 pattern may represent what we are calling hyaline fibrous involution, a conclusion substantiated by a common association with $B R C A$ mutational carrier status. Whether the presence of characteristic periacinar hyaline material reflects abnormal development vs. altered involution is unknown. However, the perimenopausal age association we observed in this study corresponds to the conclusion of physiological involution, suggesting an acquired rather than developmental etiology.

A number of recent publications implicate the importance of tumor microenvironment in tumor development and progression [18], some suggesting that DNA instability of stromal cells plays a role in carcinogenesis. Interestingly, Salem et al. [19] demonstrated that BRCAl-knock out fibroblasts induced a 2.2-fold increase in breast tumor 
growth. They addressed upregulation of HIF-1 $\alpha$, autophagy, and ketone body formation as a possible mechanism for the phenomenon. Given these findings, there might be a possible link between $B R C A$ mutation and increased collagen deposition/stromal fibrosis shown in the present study. Furthermore, mammographically dense breast tissue has been recognized as one of the greatest risk factors for developing breast carcinoma, where areas of mammographically dense breast tissue show not only increased epithelial and stromal cellularity, but also increased fibrillary collagen deposition.

Provenzano et al. [20] demonstrated that increased stromal collagen in mouse mammary tissue significantly increases tumor formation approximately threefold using a bi-transgenic tumor model with increased tumor stromal collagen in mouse mammary tissue, which offers some possible clues to explain why hyaline fibrous involution may be associated with increase breast cancer risk. They suggested two possible mechanisms for this phenomenon. First, increased breast density is related to a stiffer extracellular matrix conferring high local mechanical loads and higher resistance to cellular contractility for breast epithelial cells. Such changes have been shown to alter focal adhesion and Rho GTPase signaling, resulting in increased proliferation and phenotypic transformation of epithelial cells. Second, stromal fibroblasts may regulate epithelial cells in part through secretion of specific soluble growth factors and chemokines such as TGF-beta, EFGR, HER2, and insulinlike growth factors.

To our knowledge, this is the first paper to quantify presence of hyaline fibrous involution. Two papers mentioned above [16, 17] included photomicrographs showing strikingly similar histologic features to hyaline fibrous involution, but they did not describe histologic findings in detail to characterize this as a new entity. Also, it could be argued that hyaline fibrous involution resembles so called hyaline sclerosing lobular hyperplasia (fibroadenomatoid mastopathy). Hyaline sclerosing lobular hyperplasia is found in breast tissue surrounding about $50 \%$ of fibroadenomas and most phyllodes tumors. However, fibroadenomatoid mastopathy and hyaline fibrous involution are distinct; fibroadenomatoid mastopathy is characterized by enlarged lobules composed of an increased number of acini, whereas hyaline fibrous involution lobules are smaller and contain fewer acini than normal lobules.

Based on our findings, we further theorize that hyaline fibrous involution might reflect a form of pathologic involution, possibly caused by or associated with cellular injury. This would account for its more frequent but not exclusive presence in the setting of BRCA mutation. Therefore, we hypothesize that hyaline fibrous involution may represent a previously uncharacterized, preneoplastic benign terminal duct lobular unit phenotype associated with an increased risk of breast cancer regardless of $B R C A$ mutation status. More studies involving larger cohorts and ancillary studies will be required.

\section{Compliance with ethical standards}

Conflict of interest The authors declare that they have no conflict of interest.

Publisher's note: Springer Nature remains neutral with regard to jurisdictional claims in published maps and institutional affiliations.

\section{References}

1. Milanese TR, Hartmann LC, Sellers TA, Frost MH, Vierkant RA, Maloney SD, et al. Age-related lobular involution and risk of breast cancer. J Natl Cancer Inst. 2006;98:1600-7.

2. Radisky DC, Hartmann LC. Mammary involution and breast cancer risk: transgenic models and clinical studies. J Mammary Gland Biol Neoplasia. 2009;14:181-91.

3. Radisky DC, Visscher DW, Frank RD, Vierkant RA, Winham S, Stallings-Mann M, et al. Natural history of age-related lobular involution and impact on breast cancer risk. Breast Cancer Res Treat. 2016;155:423-30.

4. Golgar D, Devilee P. Genetic susceptibility: inherited syndromes. BRCA1 and BRCA2 syndromes. In: Lakhani SR, Schnitt SJ, Tan $\mathrm{PH}$, van de Vijver MJ, editors. WHO classification of tumours of the breast. 4th edn. Lyon, France: International Agency for Research on Cancer; 2012. p. 176-82.

5. Shiovitz S, Korde LA. Genetics of breast cancer: a topic in evolution. Ann Oncol. 2015;26:1291-9.

6. Venkitaraman AR. Linking the cellular functions of BRCA genes to cancer pathogenesis and treatment. Annu Rev Pathol. 2009;4:461-87.

7. Hartmann LC, Lindor NM. The role of risk-reducing surgery in hereditary breast and ovarian cancer. N Engl J Med. 2016; 374:454-68.

8. Hartmann LC, Sellers TA, Frost MH, Lingle WL, Degnim AC, Ghosh K, et al. Benign breast disease and the risk of breast cancer. N Engl J Med. 2005;353:229-37.

9. Venables WN, Ripley BD. Modern Applied Statistics with S. 4th edn. New York: Springer; 2002. p. 206-7.

10. Gray B. cmprsk: Subdistribution analysis of competing risks. $\mathrm{R}$ package version 2.2-6. 2013. http://CRAN.R-project.org/packa $\mathrm{ge}=$ cmprsk.

11. Hoogerbrugge N, Bult P, de Widt-Levert LM, Beex LV, Kiemeney LA, Ligtenberg MJ, et al. High prevalence of premalignant lesions in prophylactically removed breasts from women at hereditary risk for breast cancer. J Clin Oncol. 2003;21:41-5.

12. Leunen K, Drijkoningen M, Neven P, Christiaens MR, Van Ongeval C, Legius E, et al. Prophylactic mastectomy in familial breast carcinoma. What do the pathologic findings learn us? Breast Cancer Res Treat. 2008;107:79-86.

13. Kauff ND, Brogi E, Scheuer L, Pathak DR, Borgen PI, Hudis CA, et al. Epithelial lesions in prophylactic mastectomy specimens from women with BRCA mutations. Cancer. 2003; 97:1601-8.

14. Arun B, Vogel KJ, Lopez A, Hernandez M, Atchley D, Broglio $\mathrm{KR}$, et al. High prevalence of preinvasive lesions adjacent to BRCA1/2-associated breast cancers. Cancer Prev Res (Phila). 2009;2:122-7. 
15. Adem C, Reynolds C, Soderberg CL, Slezak JM, McDonnell SK, Sebo TJ, et al. Pathologic characteristics of breast parenchyma in patients with hereditary breast carcinoma, including BRCA1 and BRCA2 mutation carriers. Cancer. 2003;97:1-11.

16. Bayraktar S, Qiu H, Liu D, Shen Y, Gutierrez-Barrera AM, Arun $\mathrm{BK}$, et al. Histopathological features of non-neoplastic breast parenchyma do not predict BRCA mutation status of patients with invasive breast cancer. Biomark Cancer. 2015;7:39-9.

17. Russo J, Lynch H, Russo IH. Mammary gland architecture as a determining factor in the susceptibility of the human breast to cancer. Breast J. 2001;7:278-91.
18. McCready J, Arendt LM, Rudnick JA, Kuperwasser C. The contribution of dynamic stromal remodeling during mammary development to breast carcinogenesis. Breast Cancer Res. 2010; 12:205.

19. Salem AF, Howell A, Sartini M, Sotgia F, Lisanti MP. Downregulation of stromal BRCA1 drives breast cancer tumor growth via upregulation of HIF-1alpha, autophagy and ketone body production. Cell Cycle. 2012;11:4167-73.

20. Provenzano PP, Inman DR, Eliceiri KW, Knittel JG, Yan L, Rueden CT, et al. Collagen density promotes mammary tumor initiation and progression. BMC Med. 2008;6:11. 